\section{Una aproximación a las discusiones en el campo de la educación y la pedagogía: Estado de la cuestión}

\author{
An Approach to the Discussions \\ on Education and Pedagogy: \\ State of the Art
}

Uma abordagem das discussões

no campo da educação e da pedagogia: Estado da questão

\section{Ana Cristina León Palencia*}

\section{Resumen}

El presente artículo es una reflexión derivada de una investigación que intenta elaborar un mapa de las discusiones sobre el campo de la educación y la pedagogía. Para ello, retoma algunas de las clasificaciones elaboradas a partir de conceptos como corrientes, tendencias, enfoques o tradiciones pedagógicas. Con base en esta última noción y en sus desarrollos —articulada a los conceptos de educación, formación y currículo- se propone el diseño de una nueva cartografía del campo. Para finalizar, se indican algunos desarrollos del estado de la discusión en Colombia y la necesidad de construir rutas de trabajo que posibiliten actualizar los estudios de este campo en el país.

\section{Palabras clave}

Pedagogía; educación; formación; tradiciones pedagógicas

\section{Abstract}

This reflection paper is a product of a research aimed at making a map of discussions in the field of education and pedagogy. For that purpose, it takes up again some classifications developed from concepts such as currents, trends, approaches and pedagogical traditions. Focusing on the latter and its developments - connected with the concepts of education, bildung and curriculum - a new cartography is proposed the design of a new cartography. Finally, the paper elaborates on some elements of the current discussion in Colombia and the need for new paths to bring up to date the field of pedagogical studies in the country.

\section{Keywords}

Pedagogy; education; bildung; pedagogical traditions

\section{Resumo}

0 presente artigo é uma reflexão derivada de uma pesquisa que procura elaborar um mapa das discussões sobre o campo da educação e da pedagogia. Para isso, retomam-se algumas das classificações que foram desenvolvidas a partir de conceitos tais como correntes, tendências, abordagens e tradições pedagógicas. Baseando-se na última noção e nos seus desenvolvimentos - articulada aos conceptos de educação, formação e currículo- propõe-se o design de uma nova cartografia do campo. Finalmente, com base em alguns desenvolvimentos da situação na Colômbia, observa-se a necessidade de construir rotas de trabalho que permitam a atualização desse campo de estudos no país.

\section{Palavras-chave}

Pedagogia; educação; formação; tradições pedagógicas

Fecha de recepción: octubre 14 de 2015 Fecha de aprobación: abril 12 de 2016

................................................ 


\section{Introducción}

$\mathrm{E}$ n el campo pedagógico, abordar la idea de discusiones, enfoques, escuelas de pensamiento, teorías, tendencias o corrientes es un ejercicio que alude a una clasificación disímil y que difícilmente cuenta con un consenso. Por este motivo, antes que presentar una apuesta de ese orden es necesario señalar de qué se habla cuando se espera establecer una filiación a alguna de dichas clasificaciones. En este sentido, es importante recordar que toda clasificación es un asunto caprichoso, que busca incluir, excluir y diferenciar. Cuando se estudia o se elige una forma de clasificación, una clave para hacer tal selección es identificar el criterio que se usa para agrupar unas características y designarlas bajo un nombre (o viceversa). Siguiendo estas premisas, a continuación se presentarán algunas maneras en las que se han descrito y organizado las discusiones pedagógicas contemporáneas para, posteriormente, señalar la perspectiva en que se instalan estos análisis.

En primer lugar, para Canfux et al. (2000) la noción de tendencia pedagógica tiene que ver con aquellos discursos que cuentan con un cierto nivel de sistematización y que, a partir de una concepción de hombre, han elaborado una propuesta acerca de "la educación, sus protagonistas, el proceso de enseñanza-aprendizaje, sus finalidades y su modo de realización" (p.17). Dichas ideas se habrían configurado bajo la mediación de factores socio-históricos, entre los que se contemplan aspectos económicos, políticos, ideológicos y del desarrollo de la técnica y las ciencias afines al campo de la educación. De este modo, serían tendencias pedagógicas contemporáneas las denominadas: pedagogía tradicional, escuela nueva, tecnología educativa y el uso de las NTIC en la educación, la pedagogía autogestionaria, la pedagogía no directiva, pero también en la enseñanza centrada en el estudiante, la pedagogía liberadora, la perspectiva cognoscitiva, Jean Piaget y la pedagogía operatoria, el constructivismo, la investigación-acción, la teoría crítica de la enseñanza y el enfoque histórico-cultural. Desde esta mirada, es posible visibilizar la multiplicidad de discursos que se agencian sobre y desde el escenario pedagógico, pero además, la manera como aplica una clasificación, traslapando discursos, creando otros, acopiando saberes de múltiples áreas y complejizando la identificación de la noción de pedagogía y educación que atraviesa tal clasificación.

Un segundo caso, a partir de la misma noción pero para el contexto colombiano, lo encontramos en Tamayo (2007), quien define las tendencias de la pedagogía en Colombia a partir de los conceptos que nuclean las discusiones sobre el asunto en el país. Para ello, retoma los planteamientos de Díaz (1993) a propósito del campo intelectual de la educación y describe las condiciones que en la década de los ochenta ${ }^{1}$ posibilitaron comprender la pedagogía, por lo menos de cuatro formas: (1) como dispositivo, configurada por Mario Díaz, quien, siguiendo los desarrollos de la sociolingüística de Bernstein, concibe la pedagogía como un dispositivo que regula los discursos, significados y prácticas en los procesos de transformación de la cultura; (2) como disciplina, en la cual se siguen los planteamientos de Olga Lucía Zuluaga (1999), para quien la pedagogía es la disciplina que "conceptualiza, aplica y experimenta los conocimientos referentes a la enseñanza de los saberes específicos en las diferentes culturas" (p. 144); (3) como disciplina reconstructiva, noción desarrollada por el grupo Federici (Universidad Nacional), en la que se asume que la pedagogía "pretende transformar un saber-cómo, domeñado prácticamente en un saber-qué explícito" (Mockus, 1995, p. 18). En esta tendencia se interroga la relación entre el conocimiento escolar y el extraescolar, buscando su articulación a través de la idea de juegos de lenguaje retomada de Ludwig Wittgenstein. A su vez, apoyados en Habermas,

se insiste en que la ciencia y la técnica no constituyen la única esfera de racionalidad desarrollada por la modernidad. También el derecho, la moral, la crítica artística pueden ser y han sido terrenos de una argumentación racional elaborada, decantada por escrito y relevante para ciertas órbitas de la acción. (p. 71)

Finalmente, (4) la pedagogía desde el constructivismo, que sería más una posición epistemológica con consecuencias didácticas que una pedagogía propiamente, pero a partir de la cual -según el autor-sería posible construir un campo de saber al rastrear las prácticas de los maestros, sus métodos, procesos de aprendizaje, conocimiento escolar, desarrollo cognitivo y afectivo, axiológico y estético, como posibilidad de trabajo sobre la enseñanza.

Un tercer caso lo presenta Suárez (2000) a partir de la noción de corrientes pedagógicas, desde dos consideraciones: la primera, en su comprensión

1 Condiciones como: "el Movimiento Pedagógico, la nueva legislación educativa, el auge de los postgrados en educación, la profusión de publicaciones en educación y pedagogía, la consolidación de Centros de investigación en universidades públicas y privadas, las políticas del Instituto Colombiano para el fomento de la Educación Superior (Icfes) y el Programa de estudios Científicos en Educación y Pedagogía en Colciencias además de los cambios paradigmáticos en cuanto al objeto y método de las Ciencias Sociales" (Tamayo, 2007, p. 66). 
como campo o discurso, que expresa líneas de fuerza en el pensamiento o en la práctica educativa; y una segunda comprensión, como los movimientos o teorías caracterizados por tener una línea de pensamiento e investigación definida, a propósito de la cual se realizan aportes permanentes que proporcionan coherencia, solidez y existencia en el tiempo a los discursos que la constituyen. Dichas corrientes

describen, explican, conducen y permiten la comprensión de lo pedagógico ante las exigencias del contexto y pasan a ser referentes que crean y recrean los contextos sociales y pedagógicos de la escuela y/o las líneas de discurso o de la práctica. (p. 42)

Para dicho autor, serían entonces tres las corrientes pedagógicas contemporáneas:

a. el paradigma ecológico, en el cual la escuela se lee como un ecosistema social humano, que expresa la realidad desde un complejo entramado de elementos (población, ambiente, interrelaciones y tecnología) y de relaciones organizativas que la configuran y determinan como tal;

b. las pedagogías críticas, que si bien obedecen a una amplia gama de enfoques teóricos relativos a los procesos sociales, culturales, políticos, educativos, de la escuela, el hombre y la sociedad, se originan en las teorías críticas derivadas de Escuela de Frankfurt y se concentran en por lo menos cuatro relaciones: educación-escuela-sociedad, conocimiento-poder-subjetivación, institucionalización-hegemonía y los vínculos entre teoría y práctica. Por último,

c. el constructivismo, que siguiendo a GallegoBadillo (1996), se entiende como una estructura conceptual, metodológica y actitudinal, en la cual se conjugan teorías de la psicología cognitiva, la epistemología, la lógica, la lingüística, la pedagogía y la didáctica, a propósito de la pregunta por cómo aprende el hombre. Para ello, se aborda la relación entre desarrollo y aprendizaje. Esta segunda perspectiva, quizá más concreta, llama la atención por la variedad de disciplinas que se ponen en juego, pero también por los múltiples criterios de clasificación, bien sea desde propuestas de lectura de lo educativo, o desde preguntas por el aprendizaje.

d. Un cuarto y último caso es el de Marco Raúl Mejía (2006), quien para abordar los discursos de la educación popular en Colombia desarrolla una interesante red conceptual en la que vincula y diferencia las nociones de paradigma, corriente, enfoque y línea metodológica, de manera jerárquica e interrelacionada. La noción principal sería — según Mejía (2006) - la de paradigma, que está constituido por una tradición educativa, descrita por sus condiciones culturales, políticas, económicas y lingüísticas, a través de las cuales se generan representaciones del mundo, del sí mismo y los otros, en síntesis, del proyecto de ser humano. Históricamente, se habrían configurado cuatro paradigmas: el alemán, el francés, el sajón (o anglo-americano) y el latinoamericano.

En estas tradiciones "paradigmáticas" se desarrollan corrientes que definen el horizonte social y los intereses de la acción educativa. Estas, con base en una mirada de corte más conceptual y teórico, realizan dichas definiciones desde lecturas de mundo particulares, que ubican la pregunta por el nexo educación-escuela-pedagogía-proyecto institucional, con el destino y el futuro de la sociedad. Entonces, en cada paradigma se ubicarían luchas de diferentes grupos, sectores y concepciones, por redefinir los sentidos de la educación. En esta línea se plantean tres corrientes que reconfigurarían los paradigmas de manera permanente: la corriente clásica-tradicional, que se establece desde la fuerza del pasado; la corriente modernizadora-renovadora, que considera que la educación debe hacer actualizaciones que respondan a las necesidades de la época, y la corriente crítica-transformadora, que entiende la educación en el marco de juegos de poder.

Posterior a la identificación en la esfera de los paradigmas y las corrientes, se realiza una síntesis, que se materializa en los enfoques, da forma a los procesos pedagógicos que fundamentan conceptualmente el quehacer educativo y pedagógico, y se relacionan con asuntos como: los procesos de aprendizaje, sus mediaciones, herramientas y las interacciones entre adultos y las generaciones más jóvenes. Al decir de Mejía (2006), podrían identificarse tres grandes enfoques iniciales que se constituyeron entre la segunda mitad del siglo XIX y la primera mitad del siglo xx: la pedagogía frontal o transmisionista, la pedagogía activa o nueva y la pedagogía de la tecnología educativa. A su vez, se habrían recreado a lo largo del siglo xx otros enfoques, como el constructivista piagetiano, el socio-histórico de Vigotsky y el enfoque crítico liberador.

Por último, el desarrollo metodológico exigiría de quienes lo realizan una ruta concreta en la cual los principios pedagógicos enunciados bajo la forma de enseñanza-aprendizaje se concreten a 
través de herramientas didácticas, enunciados de lenguaje, sentidos, etc., que se definen por el o los enfoques que se asuman en la práctica y dan lugar a las denominadas líneas metodológicas. Dicha noción, que concluye el mapa construido por Mejía (2006), opera a través de la enseñanza-aprendizaje, que sería posible de múltiples formas: conceptual, integradora, problematizadora, contextualizada, por indagación, significativa, colaborativa, por modificabilidad cognitiva, por competencias, por diferenciación, por comprensión, entre otras.
Como se evidencia, los modos de organizar los discursos pedagógicos son diversos ${ }^{2}$, desde las tendencias pedagógicas, definidas bien sea por el nivel de sistematización y su carácter reciente (Cafux et al., 2000), o por sus conceptos nodales (Tamayo, 2007), o a través de la idea de corrientes como líneas de pensamiento o investigación en permanente construcción (Suárez, 2000) o finalmente, mediante la jerarquización de conceptos que van del general al específico, iniciando con el paradigma como la sombrilla que acoge a corrientes y enfoques y que se materializan en propuestas metodológicas definidas como líneas (Mejía, 2006).

En el cuadro 1 se sintetizan las clasificaciones antes descritas:

Cuadro 1. Síntesis de clasificaciones del discurso educativo y pedagógico

\begin{tabular}{|c|c|c|c|c|}
\hline Clasificación & Tendencia pedagógica & $\begin{array}{l}\text { Tendencias de } \\
\text { la pedagogía } \\
\text { en Colombia }\end{array}$ & $\begin{array}{l}\text { Corrientes } \\
\text { pedagógicas }\end{array}$ & Paradigma \\
\hline Criterio & $\begin{array}{l}\text { Nivel de sistematización } \\
\text { y carácter reciente }\end{array}$ & $\begin{array}{l}\text { Conceptos } \\
\text { nucleadores }\end{array}$ & \begin{tabular}{|l} 
Líneas de \\
pensamiento o \\
investigación \\
en permanente \\
construcción
\end{tabular} & $\begin{array}{l}\text { Condiciones } \\
\text { culturales, políticas, } \\
\text { económicas y } \\
\text { lingüísticas, a } \\
\text { través de las } \\
\text { cuales se generan } \\
\text { representaciones } \\
\text { del mundo, del sí } \\
\text { mismo y los otros, en } \\
\text { síntesis, del proyecto } \\
\text { de ser humano } \\
\end{array}$ \\
\hline Autores & (Cafux et. al., 2000) & (Tamayo, 2007) & (Suárez, 2000) & (Mejía, 2006) \\
\hline $\begin{array}{l}\text { Tipos del } \\
\text { discurso } \\
\text { pedagógico }\end{array}$ & $\begin{array}{l}\text { 1. Pedagogía tradicional } \\
\text { 2. Escuela nueva } \\
\text { 3. Tecnología educativa y el } \\
\text { uso de las NTic en la educación } \\
\text { 4. Pedagogía autogestionaria } \\
\text { 5. Pedagogía no directiva } \\
\text { 6. Enseñanza centrada } \\
\text { en el estudiante } \\
\text { 7. Pedagogía liberadora } \\
\text { 8. Perspectiva cognoscitiva } \\
\text { 9. Jean Piaget y la } \\
\text { pedagogía operatoria } \\
\text { 10. El constructivismo } \\
\text { 11. La investigación-acción } \\
\text { 12. La teoría crítica } \\
\text { de la enseñanza } \\
\text { 13. El enfoque } \\
\text { histórico-cultural }\end{array}$ & $\begin{array}{l}\text { 1. Pedagogía } \\
\text { como dispositivo } \\
\text { (Mario Díaz) } \\
\text { 2. La pedagogía como } \\
\text { disciplina, (Olga Lucía } \\
\text { Zuluaga, 1999) } \\
\text { 3. La pedagogía } \\
\text { como disciplina } \\
\text { reconstructiva, } \\
\text { (Grupo Federici, } \\
\text { Universidad } \\
\text { Nacional) } \\
\text { 4. La pedagogía desde } \\
\text { el constructivismo. }\end{array}$ & $\begin{array}{l}\text { 1. El paradigma } \\
\text { ecológico } \\
\text { 2. Las pedagogías } \\
\text { críticas } \\
\text { 3. El constructivismo }\end{array}$ & $\begin{array}{l}\text { 1. Tradiciones } \\
\text { pedagógicas } \\
\text { 2. Corrientes } \\
\text { pedagógicas } \\
\text { 3. Enfoques } \\
\text { 4. Líneas } \\
\text { metodológicas }\end{array}$ \\
\hline
\end{tabular}

2 Aunque en ocasiones sea más acertado señalar que dicha organización no obedece a ningún criterio, por cuanto tales discursos no son "excluyentes", es decir, no hay clasificación, como lo mencionaba Foucault (1998) siguiendo a Borges, al recordar cierta enciclo- 


\section{Un nuevo intento de clasificación: las tradiciones pedagógicas}

Ahora bien, dado que no es posible eludir el optar por una perspectiva de clasificación -en especial para quien se interesa por el estudio de este campo-, esta será entendida como una posibilidad de identificar las reglas de razonamiento y en ese sentido la que considero más interesante - por ofrecer un panorama pedagógico- es la de: tradiciones pedagógicas, siguiendo en ello a Popkewitz (2003), para quien esta estrategia es una posibilidad de problematizar los convencionalismos de la "razón" que cierran el presente y se describen desde la innovación. Para ello, opera realizando un "inventario de las diferentes trayectorias para diagnosticar su sistema de razón" (p. 148), e identificar las maneras en que se han configurado sujetos, prácticas sociales e instituciones (educativas), reconociendo que su emergencia da cuenta de la interconexión con una multiplicidad de procesos históricos.

En esta misma lógica, Noguera (2012) retoma a Popkewitz y a Schriewer para hablar de tradiciones pedagógicas. Del primero recoge y amplía su noción de "epistemología social", entendida como la "relación del conocimiento, en tanto campo cultural de prácticas que se interrelacionan con fenómenos sociales en la producción de los principios de acción y práctica" (Popkewitz, 2003, p. 176), que en otras palabras - siguiendo a Noguera-, tendría que ver con cómo los "sistemas de ideas construyen, configuran y coordinan prácticas a partir de sus principios" (Noguera, 2012, p. 210). De Schriewer retoma que, además de los fenómenos político-sociales y los referentes intelectuales diversos, las tradiciones intelectuales serían producto de "selecciones epistemológicas y exclusiones intelectuales, que mientras favorecen unas opciones teóricas, impiden otras" (Schriewer, 2000, citado en Noguera, 2012, p. 210).

En tal sentido, a continuación se abordarán brevemente a manera de inventario ${ }^{3}$, los planteamientos frente a tres tradiciones pedagógicas descritas por Noguera (2012) a partir de lo que denomina un mapa

pedia en la que los animales se dividían en: "a) pertenecientes al Emperador, b) embalsamados, c) amaestrados, d) lechones, e) sirenas, f) fabulosos, g) perros sueltos, h) incluidos en esta clasificación, i) que se agitan como locos, j) innumerables, k) dibujados con un pincel finísimo de pelo de camello, l) etcétera, m) que acaban de romper el jarrón, n) que de lejos parecen moscas" (p. 1).

3 Dadas las características de este documento, solo se señalarán algunos puntos para la discusión, a propósito de las teorías pedagógicas "contemporáneas”. conceptual. Este constituye el horizonte epistémico de algunos de los abordajes "recientes" ${ }^{\text {"vinculados }}$ a dichas tradiciones (que se señalarán brevemente), así como de algunos debates generados en el panorama colombiano. Entonces, desde este análisis se reconoce la necesidad de comprender los vínculos históricos de algunos planteamientos "recientes", a partir de un mapa conceptual, relativo a tres tradiciones pedagógicas: la francófona, a través del concepto de educación y el desarrollo de las ciencias de la educación; la germánica y la noción de "bildung" o formación, y la anglosajona desde el concepto de currículo; así mismo, se pretende puntualizar algunos de los discursos sobre este asunto en Colombia.

Para comenzar, es necesario aclarar que el mapa conceptual que se describirá a continuación no intenta desarrollar en sentido general cada una de las tradiciones -cuestión que excede las pretensiones de este documento-. Por el contrario, busca señalar la tradición en la que se instalaría cierto discurso, entendiendo una suerte de "pluralidad teórico-disciplinaria" (Runge, 2008, p. 25) en la que ninguna tradición gozaría desde su especificidad, de mayor reconocimiento que otras. De igual forma, es necesario precisar que las tradiciones emergieron en la modernidad y dieron lugar justamente al discurso pedagógico, y que se trata de identificar qué nociones emergieron, se vincularon y operaron en una determinada tradición intelectual (Noguera, 2014).

Una primera tradición será la francófona, establecida en torno al concepto de "educación" y cuyos desarrollos fueron posibles a través de las ciencias de la educación. Uno de los referentes de dicho concepto es el planteamiento de J. J. Rousseau (1762) en su libro el Emilio o de la educación, en el que pone en juego ideas como naturaleza e interés y que, posteriormente, fueron apropiados por los pedagogos activos (durante el siglo $\mathrm{xx}$ ) en sus múltiples versiones. Por una parte, desde esta tradición se reconocen vínculos directos con disciplinas como la sociología y la psicología (Durkheim, 1911) ${ }^{5}$, cuya centralidad

4 Distanciándose de ciertas lecturas sobre lo contemporáneo instaladas en la novedad, desdibujando la lectura histórica y desvinculándose de la tradición. En este sentido, Agamben (2008) señalaba al contemporáneo como: "aquel que no se deja cegar por las luces del siglo y que logra distinguir en ellas la parte de la sombra, su íntima oscuridad" y que "percibe la oscuridad de su tiempo como algo que le corresponde y no deja de interpelarlo, algo que, más que otra luz se dirige directa y especialmente a él. Contemporáneo es aquel que recibe en pleno rostro el haz de tinieblas que proviene de su tiempo" (s. p.).

5 Para el caso colombiano, puede revisarse el trabajo de Ríos (2007), a propósito de las ciencias de la educación en Colombia, en el cual, se describen dos ejes emergentes durante el 
ha sido puesta en cuestión, pues en ocasiones ha mirado el campo educativo como uno más entre otros escenarios prácticos en los que se despliegan sus teorías, y en el que la pedagogía no ha constituido un lugar de reflexión, ya que como lo señalan Sáenz et al. (2010) sus desarrollos han estado marcados por la precariedad, fragmentación e ilegitimidad, en el caso francés, fundamentalmente debido a tres factores:

En primer lugar, (a) la relativa hegemonía que han ejercido las disciplinas enseñadas en términos de definir sus propias formas de enseñanza, especialmente en la educación secundaria. En segunda instancia, con la relativa y débil apropiación de las ciencias humanas en la educación francesa. En tercer lugar, con el carácter conservador, centralista y uniformador del sistema público de educación francés que ha sido un obstáculo formidable para la innovación. (p. 57)

Por otra parte, la introducción de las ciencias de la educación en el escenario universitario (entre otros por Chateau, Debesse y Mialaret en 1967) posibilitó el reconocimiento de una serie de campos de discusión educativa, que si bien no todos lograron legitimidad, e incluso existieron en detrimento de la "pedagogía", permitieron ampliar la lectura de lo educativo en por lo menos tres ramas: a) las disciplinas que estudian las condiciones generales y locales de la educación (historia de la educación y la pedagogía, sociología de la educación, demografía escolar, economía de la educación y educación comparada); b) las ciencias que estudian las situaciones y los hechos educativos (disciplinas que estudian el acto educativo, ciencias de las didácticas y teorías de los programas, ciencias de los métodos y de las técnicas y ciencia de la evaluación); y por último, c) las ciencias de la reflexión y del futuro (la filosofía de la educación y las ciencias que miran al futuro - la planificación educativa-) (Mialaret, 1981). Como se evidencia, buena parte de estas disciplinas no fueron cobijadas bajo el halo científico, lo que en cierta medida puso en duda su legitimidad.

De esta tradición devienen planteamientos recientes como los de Philippe Meirieu, intelectual francés que busca reivindicar el lugar de la pedagogía en las discusiones educativas en contraposición a las ciencias de la educación. De este modo, en trabajos como el de Emilio, vuelve pronto... iSe han vuelto locos! (Meirieu y Develay, 2003), se plantea la necesidad de reconocer la pedagogía como una disciplina que tiene su propio vocabulario, que es necesaria en

proceso de institucionalización de las mismas en el país, un eje sociológico, con dos vertientes, la del determinismo biológico y el relativismo cultural, y un eje psicológico, en dos vías, desde una psicología racional y una psicología experimental. la formación de especialistas de la educación, que tiene sus propias problemáticas. En suma, se plantea la necesidad de una "democratización del sistema educativo" (p. 53). Para Meirieu (2007), entonces, la pedagogía consiste en idear

condiciones que posibiliten compartir saberes, el goce de descubrirlos, la felicidad de sentirse en condiciones de hacer propia la herencia de los hombres, prolongarla y superarla. La pedagogía debe proseguir e intensificar sus investigaciones y trabajos en ámbitos que, en esos terrenos, siguen en gran medida inexplorados: las relaciones entre los aprendizajes cognitivos y la socialización, las condiciones que favorecen la transferencia de conocimientos, los obstáculos de todo orden que bloquean el acceso a los saberes. (p. 139)

En Colombia, Ríos (2006) describe un escenario semejante frente a la discusión a propósito de la pedagogía, pues en los años ochenta se produjo el florecimiento de una serie de discursos sobre el tema, a través de trabajos como el de Olga Lucía Zuluaga o del grupo Federici ${ }^{6}$. Sin embargo, para dicho autor la implementación en el país del Decreto 1278 del 2002, según el cual profesionales no licenciados pueden ejercer el cargo de docentes tras certificar un curso de pedagogía y acreditar un periodo de prueba, desplaza el lugar de la pedagogía, otrora central en la formación de maestros.

Como segunda tradición se identifica la germánica, descrita a través del concepto de Bildung, o formación, vinculado con la filosofía y que encuentra en los planteamientos del pedagogo alemán Herbart una suerte de sistematicidad y de concreción mediante la pedagogía general. Una de las particularidades de esta tradición tiene que ver con la denominación de la pedagogía como "ciencia de la educación", en singular. Este aspecto visibiliza una lectura de la pedagogía como "campo disciplinar de estudio e investigación" (Runge, 2008, p. 30), un campo "plural y abierto", que tiene por objeto la educación ${ }^{7}$. La coherencia de dicho campo se encontraría marcada por el acercamiento común a la educación como problema, el cual puede ser abordado desde múltiples referentes teóricoconceptuales. En esta tradición se pueden identificar por lo menos tres grandes troncos, siguiendo

6 Olga Lucía Zuluaga, fundadora del Grupo de Historia de la Práctica Pedagógica en Colombia, grupo interuniversitario, conformado por investigadores de las universidades: de Antioquia, Nacional, Pedagógica Nacional, del Valle y Javeriana. Por otra parte, el grupo Federici emergió en la Universidad Nacional.

7 Según Runge (2008), este campo puede tomar rumbos: teórico-prácticos, aplicados, descriptivos o experimentales. 
a Runge $(2008,2010)$, a saber: el comprensivo o de las ciencias del espíritu, término propuesto para distinguir aquellas ciencias que abordan al hombre y sus producciones, se trata de una preocupación por la formación y la educación "no por el cambio social en forma general, o por el desarrollo de la psiquis en términos de aprender conocimientos" (Runge, 2001, p. 386); el empírico-experimental, que busca superar las carencias de las ciencias del espíritu, es decir, "su inocencia metodológica y su carácter no científico" (p. 388), reconocía la necesidad de implementar metodologías que describieran la realidad de modo científico; y el crítico o emancipatorio, que surge como superación de los planteamientos de las ciencias del espíritu y que con los desarrollos de la Escuela de Frankfurt instala la idea de que la "emancipación social de la humanidad debería ser vehiculizada desde la misma educación" (p. 389).

Por último, una tercera tradición se verá reflejada en los estudios derivados de la perspectiva anglosajona que tendrá por concepto central el "currículo" - el cual es preciso no confundir con el plan de estudios-, y que siguiendo a Noguera (2012) se remonta a los planteamientos de Spencer (1860), quien basado en el principio de utilidad se preguntaba por: ¿cuáles son los conocimientos más útiles? Para dar respuesta a esta inquietud identifica cinco actividades útiles para la vida humana: a) aquellas que confluyen a la preservación del individuo; b) las que contribuyen indirectamente a ella; c) las empleadas en educar y disciplinar la familia; d) las que garantizan el mantenimiento del orden social y de las relaciones políticas y e) las que ocupan los momentos de ocio y descanso (Spencer [1860], citado en Noguera [2012]). La educación será desde este discurso una práctica organizada "racionalmente" a través del principio de utilidad.

En esta lógica, se inscriben algunos de los denominados "curriculum studies", que Tomaz Tadeu da Silva $(2001)^{8}$ organiza en tres tipos de estudio: las teorías tradicionales, las críticas y las que denomina poscríticas. Para Silva, los estudios del currículum hay que reconocerlos como una construcción social, lo que deviene en desnaturalizar la idea de una "existencia" dada del currículo. Por lo tanto, un discurso "sobre el currículum, incluso cuando pretende describirlo 'tal como es', lo que hace es crear una idea particular de éste" (p. 12). Al primer grupo de estudios, definido como "teorías tradicionales", pertenecerían los desarrollos de Bobbit (1918) en su texto

8 Es necesario recordar que para Silva (2001), el currículo se equipararía a la noción de pedagogía; en esto sigue la tendencia anglosajona.
The Curriculum, que enfocado en la economía de la "eficiencia" buscaba transferir el patrón de Frederick Taylor ${ }^{9}$ al campo educativo. En esta perspectiva, la escuela debía funcionar de acuerdo a principios de la organización científica; el currículo era una cuestión de organización, por lo que las finalidades de la educación estaban dadas por las exigencias profesionales de la vida adulta. Por otra parte, Tyler (1949) a través de su modelo de organización y desarrollo debía responder a cuatro preguntas: ¿Qué objetivos educativos debe alcanzar la escuela?, ¿Qué experiencias educativas pueden tener posibilidades de que se alcancen esos propósitos?, ¿Cómo organizar eficientemente esas experiencias educativas? y ¿Cómo podemos tener la certeza de que esos objetivos se alcanzan? Dichos interrogantes suponían la división de currículo, enseñanza e instrucción, y evaluación. Sin embargo, el énfasis debía estar en la especificación de objetivos, asunto posible a través de la filosofía social y educativa y mediante la psicología del aprendizaje. Estos modelos, considerados -según Silva- "tradicionales" en el marco de los estudios del currículo, tienen énfasis tanto técnicos como progresistas de base psicológica y serán refutados a partir de los años setenta, con el llamado "movimiento de reconceptualización del currículo".

El segundo grupo, que corresponde a los trabajos de crítica sobre el currículo, se sitúa en "la actividad de garantizar un consenso, de obtener la hegemonía" (Silva, 2001, p. 17). Por ello, a diferencia de teorías tradicionales que parecen ser neutras, desinteresadas y científicas y cuyo acento es el qué, las nombradas teorías críticas y poscríticas tienen como referente la teoría sobre el poder, y el acento ya no solo estaría en el qué, sino además en el por qué. Ello implica una pregunta por el saber, la identidad y el poder. Estas teorías críticas, que emergieron en la década de los setenta del siglo $\mathrm{xx}$, se centraron en el movimiento de reconceptualización de este. Para el caso de Inglaterra, será a través de la nueva sociología de la educación, en el movimiento identificado con el sociólogo inglés Michael Young; en Brasil mediante los planteamientos de Paulo Freire, y en Francia, con los desarrollos de Althusser, Bourdieu y Passeron, Baudelot y Establet. La característica de estas teorías críticas fue la desconfianza en el statu quo, su cuestionamiento y la búsqueda de su transformación radical. Los planteamientos se instalaron aquí, para Althusser, al entender que la escuela actúa ideológicamente por medio del currículo; en Bourdieu y

9 Taylor (1856-1915) fue un ingeniero mecánico y economista estadounidense, promotor de la organización científica del trabajo. Es considerado el padre de la administración científica. 
Passeron, que la crítica radica en la noción de capital cultural. Y vía conceptos marxistas, filtrados a través de análisis contemporáneos como los de Gramsci y la escuela de Frankfurt, el énfasis estaba en el papel de las estructuras económicas y políticas en la reproducción cultural y social a través de la educación y el currículo. Una segunda ola de trabajos críticos, para Silva (2001), serán: la teoría crítica neomarxista de Michael Apple, el currículo como política cultural de Henry Giroux y los análisis de Paulo Freire, a propósito de la educación bancaria.

Por último, el tercer grupo de trabajos, los que Silva (2001) denomina poscríticos, serán aquellos que leen la escuela desde las teorías de género, étnicas, raciales, queer, poscoloniales, posestructuralistas o las derivadas de los estudios culturales. Habría que decir, siguiendo a Noguera (2012), que esta tradición, la más reciente de las anteriores, ha sido una de las más extendidas en América Latina, lo que puede evidenciarse en la pluralidad de trabajos al respecto.

En síntesis, hasta el momento se han contrastado las nociones de tendencias, corrientes y tradiciones pedagógicas, y se ha optado por ampliar esta última a la luz de sus desarrollos en las tradiciones francófona (educación-ciencias de la educación), germánica (formación) y anglosajona (currículo), las cuales permiten identificar el horizonte epistemológico o al decir de Popkewitz (2003) la "epistemología social" a la que se vinculan trabajos recientes como los de Meirieu. Por supuesto, hay que señalar que estos son solo "puntos" de discusión, si se quisiera hablar en términos de mapa de las teorías pedagógicas, porque como lo describían Canfux et al. (2000), el listado de discursos "pedagógicos contemporáneos" es múltiple y corresponde a naturalezas analíticas distintas.

\section{Discusiones sobre la educación y la pedagogía en Colombia: ¿cómo clasificarlas?}

Para finalizar, se indicarán algunos de los debates sobre la educación y la pedagogía en el terreno colombiano, que podrían instalarse como "referentes" de la discusión en este campo. Por una parte, se reconoce como cantera discursiva la década de los ochenta, que a la luz de lo que se nomino "Movimiento Pedagógico colombiano" ${ }^{10}$, posibilitó la emergencia

10 El llamado Movimiento Pedagógico en Colombia surgió en 1982 y se constituyó en un acontecimiento importante gestado por el magisterio, intelectuales y sindicalistas. Para Marco Raúl Mejía el Movimiento Pedagógico surgió de la confluencia de cuatro procesos históricos: (1) La reforma curricular que se pretendía imponer por parte del Ministerio de de una variedad de discursos cuyos referentes diversos han sido y continúan siendo objeto de análisis. Si bien no podríamos hablar de una tradición, ni comunidad en este campo en el país (Runge, 2012a), sí podemos identificar el florecimiento de debates al respecto. En particular, pueden señalarse tres líneas fuertes de la discusión ${ }^{11}$ : la primera, identificada en los trabajos de Mario Díaz (1993) sobre el campo intelectual de la educación y su dispositivo pedagógico (siguiendo en ello a Basil Bernstein); la segunda, el planteamiento del grupo Federici y sus desarrollos de una pedagogía reconstructiva, de la mano de la propuesta habermasiana; y la tercera línea, las investigaciones de los miembros del Grupo de Historia de la Práctica Pedagógica en Colombia que a la luz de algunas nociones del filósofo francés Michel Foucault construyen una manera particular de leer la pedagogía, a través de categorías como saber pedagógico o práctica pedagógica.

En la primera línea, el discurso pedagógico es para Mario Díaz (1993) "una modalidad de comunicación especializada mediante la cual la transmisión/ adquisición (aprendizaje) se ve afectada. El discurso pedagógico produce límites al discurso. En este sentido es un medio de recontextualización o reformulación de un discurso primario" (p. 221). Se trata entonces de un proceso de reproducción de discursos selectivamente limitados, reorganizados, distribuidos y reubicados en el campo de la reproducción discursiva. De esta tarea se ocupa el discurso pedagógico, que transforma el discurso primario (derivado del campo de producción discursiva) en un discurso secundario (el campo de reproducción discursiva) que actúa como principio de recontextualización.

En la segunda, el grupo Federici ${ }^{12}$ define la pedagogía como una disciplina reconstructiva, que pretende transformar un saber-cómo (domeñado), en un saber-qué (explícito) "es una disciplina falible, cuyas formulaciones pueden ser desmentidas por contrastación con el saber-cómo domeñado en la práctica por el docente competente" (Mockus et al.,

Educación Nacional. (2) El auge de los movimientos sociales que intentaban construir proyectos alternativos. (3) La emergencia histórica de unos sujetos de pedagogía que pugnaban contra los modelos en boga y (4) Con la emergencia de un actor social colectivo que da sentido a ese quehacer.

11 Lo cual no implica el desconocimiento de otras producciones, como las de Eloísa Vasco, los trabajos etnográficos de Rodrigo Parra Sandoval, la propuesta de Rafael Flórez o los desarrollos de Carlos Eduardo Vasco.

12 Grupo formado en la Universidad Nacional, llamado así por el matemático italiano Carlo Federici. Se reconocían como miembros del grupo Antanas Mockus, Carlos Augusto Hernández, José Granés, Jorge Charum y María Clemencia Castro. 
2001, p. 19). En esta lógica, el docente debe tener una competencia comunicativa que le permita ajustar sus acciones en la búsqueda de un entendimiento mutuo y de acuerdo libre, para posibilitar la capacidad de participar en actos de habla afortunados.

Y en la tercera, el trabajo del Grupo de Historia de las Prácticas Pedagógicas (GHPP), que a partir del reconocimiento de las condiciones históricas de emergencia de la pedagogía en el país, opta por entenderla como un saber - siguiendo a Foucaulten la medida en que esta no habría alcanzado un umbral de epistemologización que le permita ser definida como ciencia o disciplina; por ello, en el campo del saber, se pueden identificar discursos sobre la escuela, el maestro, la infancia, los métodos, los saberes a enseñar, en síntesis, aquello que alude a lo pedagógico.

Estas tres líneas representan la variedad de discursos que emergieron durante la década de 1980 y que remiten a tradiciones y referentes teóricos diversos, algunos de los cuales hoy han sufrido una suerte de desdibujamiento, como es el caso de la perspectiva del Grupo Federici. Sin embargo, es preciso reconocer que durante las últimas décadas nuevas perspectivas han aparecido en el escenario nacional, algunas de ellas ligadas a la tradición del Movimiento Pedagógico y otras que se sintonizan más directamente con las tradiciones europeas. Dentro de estas vale la pena destacar las contribuciones de Runge y el Grupo Formaph ${ }^{13}$, los desarrollos sobre el "Campo conceptual de la Pedagogía" de Echeverri (2009) y las elaboraciones de distintos grupos de investigación sobre la enseñanza de las ciencias o las didácticas específicas.

Por su parte, el grupo Formaph ha concentrado sus desarrollos en la idea de la antropología histórico-pedagógica, entendida como un proyecto que "historiza y relativiza ámbitos y aspectos de lo humano, de la vida humana y de los hombres y mujeres que hasta hace muy poco se solían tener por constantes antropológicas universales" en especial, el ser humano como "Homo educandus et formabilis" (Formaph-UdeA). Así mismo, desarrollan la idea de la pedagogía como campo disciplinar y profesional (Runge, 2012b), al entenderla en su consolidación como espacio académico universitario y disciplinario, lo que implica comprenderla de dos formas, como

13 Grupo de investigación sobre Formación y Antropología Pedagógica e Histórica, de la Universidad de Antioquia. El grupo fue fundado por los profesores Jair Hernando Álvarez Torres, Juan Felipe Garcés Gómez, Diego Alejandro Muñoz Gaviria y Andrés Klaus Runge Peña. conjunto de pensamientos, reflexiones, discusiones y escritos independientes sobre la educación en su sentido amplio y en sus múltiples formas [...], - así como- en el sentido de una disciplina cuyo objeto es el conjunto de fenómenos de la educación y cuya tarea es estudiar, entender e interpretar dichos fenómenos bajo sus aspectos particulares y deslindados de otros fenómenos de la vida. (p. 7)

Esta perspectiva abre una importante discusión nacional a propósito de la formación de maestros en la medida en que permite diferenciar entre dos tipos de profesionales de la educación: el pedagogo y el maestro.

En otra perspectiva se encuentran los planteamientos recientes del profesor de la Universidad de Antioquia Alberto Echeverri (2009), a propósito del campo conceptual de la pedagogía, noción que constituye una respuesta a la perspectiva de Díaz sobre el campo intelectual de la educación, en donde la pedagogía no es más que un dispositivo de recontextualización y reproducción. Echeverri reconoce los planteamientos de

crisis, los conceptos articuladores, los proyectos reconfiguradores, el campo aplicado y el dispositivo formativo-comprensivo. El campo pedagógico recepciona e interpreta las observaciones, las experiencias, los conceptos y las teorías [...], que se constituyen, a su vez, en sus niveles de funcionamiento. Los componentes y niveles de funcionamiento son distribuidos jerárquicamente en dirección a la codificación de los elementos dispersos bajo agrupamientos conceptuales, y facilita la instalación de paradigmas de traductibilidad entre estas asimetrías. (Echeverri, 2009, p. 46)

Vale la pena mencionar una tendencia que viene en crecimiento y que no tuvo un lugar destacado durante el auge del Movimiento Pedagógico. Me refiero a las elaboraciones y los debates entre los investigadores de la enseñanza de las ciencias o de la educación en ciencias o de la didáctica de las ciencias, conceptos que son utilizados siguiendo diversas perspectivas teóricas y sobre los cuales se mantiene vigente una discusión. Si bien en un comienzo se destacaron los trabajos relacionados con la educación matemática, la enseñanza de las ciencias naturales y de las ciencias sociales, nuevos campos de reflexión e investigación se han venido consolidando como los de la enseñanza del bilingüismo, los procesos de escritura de Fabio Jurado (Castro y Henao, 2000) o la formación en ética, valores y democracia en donde cabe destacar el trabajo de Guillermo Hoyos (Castro y Henao, 2000) y de los profesores Ruiz y Quintero del Grupo Moralia. Se hace necesario un trabajo de 
investigación que permita actualizar el balance que Castro y Henao (2000) elaboraron hace más de una década para dar cuenta de manera rigurosa de las elaboraciones y discusiones de este nuevo campo de investigaciones que se ha consolidado durante las últimas décadas en el país. Por el momento, solo es posible señalarlo como uno de los acontecimientos claves en el campo de la educación y la pedagogía a nivel nacional.

Esta breve aproximación a propósito de las discusiones en el campo de la educación y la pedagogía plantea dos desafíos importantes: por una parte, señala la necesidad de renovar los balances sobre la producción de estos asuntos en nuestro país ${ }^{14}$, y por otra, expresa la necesidad de generar diversos escenarios (eventos académicos, publicaciones, etc.) en los que se reactualice el debate sobre la educación y la pedagogía, en particular, frente a sus conceptualizaciones, para así posibilitar el "campo" de la educación y la pedagogía. Como metáfora espacial prestada de Bourdieu,este existe por cuanto hay posiciones y disposiciones teóricas que actúan como líneas de fuerza que se oponen o atraen, es decir, se encuentran en tensión, y en las que están en juego los sentidos sobre lo educativo y pedagógico. Al mapear dicho campo, se pretende identificar el estado de relación de dichas fuerzas y aquello que se encuentra en juego. Y valga señalar, que si bien aquí no se explicitó una diferenciación entre las nociones de educación y pedagogía, esta continua siendo necesaria.

Finalmente, vale la pena interrogar los modos de clasificar tales debates en el país, dado que aquí el criterio de "tradición pedagógica", al parecer, no opera, ya que no está claramente definida una epistemología social o un concepto nuclear que permitan nominar una tradición pedagógica "colombiana". Sin embargo, tampoco puede incurrirse en un nihilismo, que adolece de historicidad e invisibiliza los caminos recorridos en términos de la producción educativa y pedagógica en el país (y en el mundo), generando la proliferación de "pedagogías light"15, la desprofesionalización del campo y situando como precedente una suerte de ausencia de autoridad epistemológica, según la cual cada quien define qué es pedagogía o inventa una nueva. Es justo reconocer que cuando

14 Perspectiva en la cual se adelantó el proyecto de investigación: Balance de las discusiones sobre la Pedagogía en Colombia (2000-2014) (León; Heredía, 2015), del que se deriva esta reflexión.

15 Noción acuñada por Noguera (2009), al referirse a la larga lista de nominaciones de pedagogía(s), entre las cuales podemos listar: pedagogía electoral, ciudadana, invisible, alternativa, etc. se habla de pedagogía y educación existe un canon o corpus que es preciso estudiar, quizá siguiendo más el mapa de Kan que el de Marco Polo, citando a Italo Calvino (2012) en Las ciudades invisibles:

De ahora en adelante seré yo quien describa las ciudades — había dicho el Kan-. Tú en tus viajes verificarás si existen.

Pero las ciudades visitadas por Marco Polo eran siempre distintas de las pensadas por el emperador.

- Y sin embargo he construido en mi mente un modelo de ciudad del cual se pueden deducir todas las ciudades posibles - dijo Kublai-. Encierra todo lo que responde a la norma. Como las ciudades existentes se alejan en diferente grado de la norma, me basta prever las excepciones y calcular las combinaciones más probables.

- También yo he pensado en un modelo de ciudad del cual deduzco todas las otras - respondió Marco-. Es una ciudad hecha solo de excepciones, exclusiones, contradicciones, incongruencias, contrasentidos. Si una ciudad así es absolutamente improbable, disminuyendo el número de los elementos anormales aumentan las posibilidades de que la ciudad verdaderamente exista. Por lo tanto basta que yo sustraiga excepciones a mi modelo, y de cualquier manera que proceda llegaré a encontrarme delante de una de las ciudades que, si bien siempre a modo de excepción, existen. Pero no puedo llevar mi operación más allá de ciertos límites: obtendría ciudades demasiado verosímiles para ser verdaderas. (p. 83)

Para Kan es posible definir un modo de operar de la ciudad (pedagogía) por norma, prever las excepciones y calcular combinaciones probables. En cambio, para Marco Polo, su ciudad (pedagogía), estaba hecha de excepciones, exclusiones, incongruencias y contrasentidos. Si una ciudad (pedagogía) es improbable, al disminuir las anormalidades, aumentan las probabilidades de existencia. Basta con sustraer excepciones al modelo de las ciudades (pedagogías), que incluso a modo de excepción, existirán (proliferarán pedagogías). Juzguen ustedes el mejor camino.

\section{Referencias}

Agamben, G. (2008). ¿Qué es ser contemporáneo? (Documento digital). Recuperado de http://www.ddooss.org/ articulos/textos/Giorgio_Agamben.htm

Calvino, I. (2012). Las ciudades invisibles. España: Siruela. Biblioteca Calvino. 
Canfux, V. et al. (2000). Tendencias pedagógicas en la realidad educativa actual. Colectivo de autores Cepes, Universidad de la Habana. Tarija-Bolivia: Editorial Universitaria Universidad Juan Misael Saracho.

Castro, J. y Henao, M. (comps.). (2000). Estados del arte de la investigación en educación y pedagogía en Colombia (vols. 1 y 2). Colombia: Colciencias-Socolpe.

Díaz, M. (1993). El campo intelectual de la educación en Colombia. Cali: Textos Universitarios-Universidad del Valle.

Echeverri, A. (2009). Un campo conceptual de la pedagogía: una contribución (tesis doctoral). Universidad del Valle, Colombia.

Foucault, M. (1998). Las palabras y las cosas. México: Siglo XXI.

Lenzen, D. (1985). Mito, metáfora y simulación - Perspectivas de pedagógica sistemática en la posmodernidad en la posmodernidad. En Educación (Tübingen), 38, 73-95.

Meirieu, P. (2007). Frankenstein educador. Barcelona: Laertes.

Meirieu, P. y Develay, M. (2003). Emilio, vuelve pronto... ¡Se han vuelto locos! Cali: Rudecolombia - Nueva Biblioteca Pedagógica.

Mejía, M. (2006). Educaciones y pedagogías críticas desde el Sur. (Cartografías de la educación popular). Ceaal.

Mialaret, G. (1981). Ciencias de la educación. España: Oikos-Tau.

Mockus, A. et al. (2001). Las fronteras de la escuela. Articulaciones entre conocimiento escolar y conocimiento extraescolar. Bogotá: Socolpe-Magisterio.

Noguera, C. (2012). El gobierno pedagógico. Del arte de educar a las tradiciones pedagógicas. Bogotá: Siglo del Hombre y Grupo de Historia de las Prácticas Pedagógica.

Popkewitz, T. (2003). La producción de razón y poder: historia del currículum y tradiciones intelectuales. En Popkewitz et al. (2003). Historia cultural y educación. Ensayos críticos sobre conocimiento y escolarización. Barcelona: Pomares.

Ríos, R. (2006). De la pedagogía a las ciencias de la educación: una lectura desde el saber pedagógico colombiano. Educación y Pedagogía, 18(44) 44, pp. 11-31.

Ríos, R. (2007). Las Ciencias de la educación en Colombia 1926-1954. Universalismo y particularismo cultural. Bogotá: Cooperativa Editorial Magisterio y Grupo Historia de las Prácticas Pedagógicas en Colombia.
Runge, A. (2010). Dieter Lenzen y la propuesta de una ciencia de la educación reflexiva como respuesta a la condición posmoderna. (Documento digital) Recuperado de http://es.slideshare.net/FreddyNelsonHenao/ lenzen-y-la-propuesta-de-una-ciencia-de-la-educacin-reflexiva-como-respuesta-a-la-condicin-posmoderna

Runge, A. (2008). Ensayos sobre pedagogía alemana. Bogotá: Universidad Pedagógica Nacional-Museo Pedagógico Colombiano.

Runge, A. (2012a). La pedagogía como campo intelectual: tensiones en el origen del movimiento pedagógico. Educación y Cultura, 97.

Runge, A. (2012b). Breve introducción al campo disciplinar y profesional de la pedagogía: consideraciones básicas sobre pedagogía, práctica educativa y saber pedagógico [documento de trabajo].

Runge, A. (2010). La ciencia de la educación como profesión en Alemania. En J. Sáenz (ed.). Pedagogía, saber y ciencias. Bogotá: Universidad de Antioquia, Facultad de Educación-Universidad Nacional, Facultad de Ciencias Humanas, Centro de estudios sociales (CES).

Runge, A. (2001). Panorámica general del estado actual de la ciencia de la educación en Alemania: corrientes pedagógicas, perspectivas teóricas y áreas de trabajo. En A. Echeverri. Encuentros pedagógicos transculturales: desarrollo comparado de las conceptualizaciones y experiencias pedagógicas en Colombia y Alemania (pp. 381-392). Medellín: Facultad de Educación, Universidad de Antioquia.

Sáenz, J. et al. (2010). La pedagogía en Francia: precariedad, fragmentación e ilegitimidad. En J. Sáenz (ed.). Pedagogía, saber y ciencias. Bogotá: Universidad de Antioquia, Facultad de Educación-Universidad Nacional, Facultad de Ciencias Humanas, Centro de estudios sociales (CES).

Suárez, M. (2000). Las corrientes pedagógicas contemporáneas y sus implicaciones en las tareas del docente y en el desarrollo curricular. En: Acción Pedagógica, 9,1 y 2.

Silva, T. (2001). Espacios de identidad. Nuevas visiones sobre el currículum. España: Octaedro.

Tamayo, A. (2007). Tendencias de la pedagogía en Colombia. En latinoam.estud.educ. Manizales (Colombia), $3(1), 65-76$.

Zuluaga, O. (1999). Pedagogía e historia. La historicidad de la pedagogía. La enseñanza, un objeto de saber. Colombia: Editorial Universidad de Antioquia-Anthropos-Siglo del Hombre. 